\title{
SMALL COMMUTATORS AND INVARIANT SUBSPACES
}

\author{
HUAXIN LIN
}

\begin{abstract}
Let $A$ be an operator on a Banach space, and let $T$ be a nonzero compact (polynomial compact) operator. We prove that if $T A-A T$ is "small", then $A$ has a nontrivial (hyper)invariant subspace.
\end{abstract}

Let $A$ be a nonscalar operator in $B(E)$, where $E$ is a Banach space, let $T$ be a nonzero compact operator and let $C_{T}=T A-A T$. V. I. Lomonosov, J. Daughtry, and H. W. Kim, Carl Pearcy and A. L. Shields [1-3] proved that when $C_{T}$ is very "small" (namely, has rank one or zero), $A$ has a nontrivial hyperinvariant subspace. We show here that for some other classes of operators $T$, when $C_{T}$ is very "small", $A$ has a nontrivial hyperinvariant subspace.

We need the following lemma.

LEMMA 1. Let $A \in B(E)$. If $\lambda \in \sigma(A)$, then $P(A)$ is compact, where $P(t)$ is a polynomial, and if $P(\lambda) \neq 0$, then $\lambda \in \sigma_{p}(A)$.

PROOF. Clearly $\lambda$ is on the boundary of $\sigma(A)$, hence there is $\left\{f_{n}\right\} \in E,\left\|f_{n}\right\|=1$, with $\left\|A f_{n}-f_{n}\right\| \rightarrow 0$. Hence $\left\|P(A) f_{n}-P(\lambda) f_{n}\right\| \rightarrow 0$. Since $P(A)$ is compact, we may assume that $\left\|P(A) f_{n}-f_{0}\right\| \rightarrow 0$ for some $f_{0} \in E$. Let $f_{*}=f_{0} / P(\lambda)$. Then

$$
\left\|P(\lambda) f_{n}-P(\lambda) f_{*}\right\| \leq\left\|P(\lambda) f_{n}-P(A) f_{n}\right\|+\left\|P(A) f_{n}-P(\lambda) f_{*}\right\| \rightarrow 0 .
$$

Hence

$$
\left\|P(\lambda) f_{n}-P(\lambda) f_{*}\right\| \rightarrow 0 .
$$

Since $P(\lambda) \neq 0$, we have $\left\|f_{n}-f_{n}\right\| \rightarrow 0$ and $\left\|f_{*}\right\|=1$. Hence

$$
\left\|A f_{*}-f_{*}\right\| \leq\left\|A f_{*}-A f_{*}\right\|+\left\|A f_{n}-f_{n}\right\|+\left\|f_{n}-f_{*}\right\| \rightarrow 0 .
$$

That is $A f_{*}=\lambda f_{*}$. So $\lambda \in \sigma_{p}(A)$.

THEOREM 1. Let $A \in B(E)$ be nonscalar. If there is $T \in B(E)$ with $P(T)$ compact (for some polynomial), but $\sigma(P(T)) \neq\{0\}$ such that $C_{T}=T A-A T$ has rank one, then $A$ has a nontrivial hyperinvariant subspace.

PROOF. There is $\alpha \neq 0, \alpha \in \sigma_{p}(P(T))$. Hence there is $\mu \in \sigma_{p}(T)$ such that $P(\mu)=\alpha$. Moreover, $\operatorname{ker}(T-\mu) \subset \operatorname{ker}(P(T)-P(\mu))$. Hence $0<\operatorname{dim} \operatorname{ker}(T-\mu)<$ $\infty$.

Let $\operatorname{ran}(T-\mu)^{0}$ be the annihilator of $\operatorname{ran}(T-\mu)$ in $E^{*}$. Then $\operatorname{ran}(T-\mu)^{0}=$ $\operatorname{ker}\left(T^{*}-\bar{\mu}\right)$. By Lemma $1, \bar{\mu} \in \sigma_{p}\left(T^{*}\right)$. Moreover, $\operatorname{ker}\left(T^{*}-\bar{\mu}\right) \subset \operatorname{ker}\left(\bar{P}\left(T^{*}\right)-\bar{P}(\bar{\mu})\right)$. Since $\bar{P}(\bar{\mu})=0$, we have

$$
0<\operatorname{dim} \operatorname{ker}\left(T^{*}-\bar{\mu}\right)<\infty .
$$

Received by the editors March 5, 1984 and, in revised form, January 18, 1985.

1980 Mathematics Subject Classification. Primary 47A15; Secondary 47B47. 
Since $\operatorname{dim} E / \operatorname{cl} \operatorname{ran}(T-\mu)=\operatorname{dim}\left(\operatorname{ran}(T-\mu)^{0}\right)$,

$$
0<\operatorname{dim} E / \operatorname{cl} \operatorname{ran}(T-\mu) \text {. }
$$

But $(T-\mu) A-A(T-\mu)=C_{T}$, so it follows from Theorem 1 in [3] that $A$ has a nontrivial hyperinvariant subspace.

PROPOSITION 1. Let $A \neq \lambda$ be in $B(E)$. If $T$ is a nonscalar algebraic operator in $B(E)$ such that $C_{T}=T A-A T$ has rank one or less, then $A$ has a nontrivial invariant subspace.

Proof. Let $\lambda \in \sigma_{p}(T)$ and $(T-\lambda) A-A(T-\lambda)=C_{T}$. If $\operatorname{ker}(T-\lambda) \in$ Lat $A$, there is $f_{0} \in \operatorname{ker}(T-\lambda), f_{0} \neq 0$, such that $C_{T} f_{0}=(T-\lambda) A f_{0} \neq 0$. This implies that $\operatorname{ran}\left(C_{T}\right) \subset \operatorname{ran}(T-\lambda)$. Then $A(T-\lambda)=(T-\lambda) A-C_{T}$. Hence, $\operatorname{cl} \operatorname{ran}(T-\lambda) \in \operatorname{Lat} A$. Since $A$ is nonscalar algebraic, $\operatorname{cl} \operatorname{ran}(T-\lambda)$ is a nontrivial subspace.

Let $A \neq \lambda$ be in $B(H)$ and let $T$ be a nonzero compact operator. If $C_{T}=T A-A T$ commutes with $T$, then the spectrum of $C_{T}$ becomes $\{0\}$ [4]. Under this condition, do we have the conclusion that $A$ has a nontrivial hyperinvariant subspace?

The following proposition shows that this condition is too weak to give a result.

Proposition 2. If $\operatorname{dim} E>2$, then for every $A \in B(E)$ there is a nonzero compact operator $T$ such that $C_{T}=T A-A T$ commutes with $T$.

PROOF. If $h \in E$, then since $\operatorname{dim} E>2$, there is $g \in E, g \in \operatorname{span}\{h, A h\}$. Hence we have a functional $F$, with $\operatorname{ker} F \supset \operatorname{span}\{h, A h\},\|F(g)\|=1$. Let $T f=F(f) h$ for all $f \in E$. Then $T$ is compact and $T^{2}=0$.

Now $T C_{T}-C_{T} T=T(T A-A T)-(T A-A T) T=-2 T A T$. But

$$
T A T f=F(f) T A h=F(f) F(A h) h=0 .
$$

Hence $T C_{T}-C_{T} T=0$.

The point is that when $T$ is very "small", $C_{T}$ must be very "small". So we need $T$ "larger" than $C_{T}$.

THEOREM 2. Let $A \neq \lambda$ be in $B(E)$. If there is a nonzero compact $T$ such that $C_{T}=T A-A T$ commutes with $T$ and

$$
\operatorname{Lim}_{k \rightarrow \operatorname{rank} C_{T}}\left\|T^{k}\right\|^{1 / k} \neq 0
$$

then $A$ has a nontrivial hyperinvariant subspace.

PROOF. If $\operatorname{Lim}_{k \rightarrow \infty}\left\|T^{k}\right\|^{1 / k} \neq 0$, i.e., $r(T)>0$, there is $\alpha \neq 0, \alpha \in \sigma_{p}(T)$. Hence $\bar{\alpha} \in \sigma_{p}\left(T^{*}\right)$. It is well known that

$$
\operatorname{dim} \bigvee_{n=1}^{\infty} \operatorname{ker}\left(T^{*}-\alpha\right)^{n}<\infty
$$

Hence there is an integer $k>0$ such that $\operatorname{ker}\left(T^{*}-\bar{\alpha}\right)^{k-1}=\operatorname{ker}\left(T^{*}-\bar{\alpha}\right)^{k}$ since $\operatorname{ker}\left(T^{*}-\bar{\alpha}\right)^{n} \subset \operatorname{ker}\left(T^{*}-\bar{\alpha}\right)^{n+1}$ for all $n$. In other words,

$$
\operatorname{cl}\left[\operatorname{ran}(T-\alpha)^{k-1}\right]=\operatorname{cl}\left[\operatorname{ran}(T-\alpha)^{k}\right] \text {. }
$$

Since $(T-\alpha) A-A(T-\alpha)=C_{T}$ and $T-\alpha$ commutes with $C_{T}$, by induction we have

$$
(T-\alpha)^{k} A-A(T-\alpha)^{k}=k(T-\alpha)^{k-1} C_{T} .
$$


Hence

$$
A(T-\alpha)^{k}=(T-\alpha)^{k} A-k(T-\alpha)^{k-1} C_{T} .
$$

This implies $A(T-\alpha)^{k} f \in \operatorname{ran}\left[(T-\alpha)^{k}\right]$ for all $f \in H$. Hence

$$
\operatorname{cl} \operatorname{ran}\left[(T-\alpha)^{k}\right] \in \operatorname{Lat} A \text {. }
$$

Hence $\operatorname{ker}\left[\left(T^{*}-\bar{\alpha}\right)^{k}\right] \in$ Lat $A^{*}$. But $0<\operatorname{dim}\left[\left(T^{*}-\bar{\alpha}\right)^{k}\right]<\infty$. Hence $\left.A^{*}\right|_{\operatorname{ker}\left(T^{*}-\bar{\alpha}\right)^{k}}$ has an eigenvalue $\bar{\lambda}$. But $A^{*} \neq \bar{\lambda}$. Hence $\{0\} \neq \operatorname{ker}\left(A^{*}-\bar{\lambda}\right) \neq E$. This implies that $\operatorname{cl}[\operatorname{ran}(A-\lambda)]$ is a nontrivial hyperinvariant subspace of $A$.

If $\operatorname{Lim}_{k \rightarrow \infty}\left\|T^{*}\right\|^{1 / k}=0$, the condition implies that $\operatorname{dim}\left(\operatorname{ran} C_{T}\right)=k$, with $T^{k} \neq$ 0 for some $k>0$. Since $T C_{T}=C_{T} T, \operatorname{cl}\left[\operatorname{ran} C_{T}\right] \in \operatorname{Lat} T$. But $\operatorname{dim}\left(\operatorname{ran} C_{T}\right)=k$, hence there is $\lambda \in \sigma_{p}\left(T_{\operatorname{ran} C_{T}}\right) \subset \sigma_{p}(T)$. Hence $\lambda=0$, i.e., there is $f_{0} \neq 0$, $f_{0} \in \operatorname{ran} C_{T}$ with $T f_{0}=0$. Hence $\operatorname{dim}\left[\operatorname{ran}\left(T C_{T}\right)\right] \leq k-1$.

By induction, since $T\left(T^{i} C_{T}\right)=\left(T^{i} C_{T}\right) T$, we have

$$
\operatorname{dim}\left[\operatorname{ran}\left(T^{k-1} C_{T}\right)\right] \leq 1 .
$$

On the other hand, $T^{k} A-A T^{k}=k T^{k-1} C_{T}, T^{k} \neq 0$ is compact. It follows from $[3]$ that $A$ has a nontrivial hyperinvariant subspace.

THEOREM 3. Let $A$ be a subalgebra of $B(E)$, where $E$ is a Banach space. Then Lat $A=\{0, E\}$ if and only if for all subalgebras $A^{\prime}$ of $B(E)$ with $A^{\prime} \supset A, \operatorname{rad} A^{\prime} \cap$ $C(E)=0$.

Proof. If Lat $A=\{0, E\}$, then Lat $A^{\prime}=\{0, E\}$ for every $A^{\prime} \supset A$. If $\operatorname{rad} A^{\prime} \cap$ $C(E) \neq 0$ for some $A^{\prime} \supset A$, let $A \in \operatorname{rad} A^{\prime} \cap C(E), A \neq 0$. Then for every $B \in A^{\prime}, 1-B A$ is invertible. By Lomonosov's lemma, there is a $B \in A^{\prime}$ such that $1 \in \sigma_{p}(B A)$. Thus we have a contradiction.

Conversely, suppose $E_{0} \in$ Lat $\mathcal{A}, 0 \neq E_{0} \neq E$. There is $F \in E^{*}$ such that $\|F\|=1, \operatorname{ker} F \supset E_{0}$. Let $f_{0} \in E_{0}, f_{0} \neq 0$, and define $A f=F(f) f_{0}$ for every $f \in E$. Then $A$ is a compact operator and $E_{0} \in$ Lat $A$. Let $A^{\prime}$ be the algebra generated by $A$ and $A$. Let $I=\left\{B:\left.B\right|_{E}=0, B \in A^{\prime}\right\}$. Clearly $I$ is a two sided ideal of $A^{\prime}$ and $A \in I$. Let $J$ be a maximal right ideal of $A^{\prime}$. Consider the homomorphism $\pi: A^{\prime} \rightarrow A^{\prime} / I=\bar{A}$. Then $\pi(J)$ is a right ideal of $\bar{A}$. Let $J^{\prime}$ be $\pi^{-1}(J)$. Then $J^{\prime} \supset J \cup I$ and $J^{\prime}$ is a right ideal. Hence $I \subset J$, which implies $A \subset J$, or $J^{\prime}=A^{\prime}$. If $J^{\prime}=A^{\prime}$, then $\pi(1)$ is the indentity of $\bar{A}$ which is in $\pi(J)$. Let $i \in J$ such that $\pi(i)=\pi(1)$. Then $i A \in J$. But $i A=A$, since $\pi(i)$ is the identity of $\AA$. This implies $A \in J$. Hence every maximal right ideal $J$ contains $A$. So $A \in \operatorname{rad}\left(A^{\prime}\right)$, and $\operatorname{rad}\left(A^{\prime}\right) \cap C(E) \neq 0$. This proves the theorem.

Corollary. Let $A \neq \lambda$ be in $B(E)$, where $E$ is a Banach space, and let $T$ be a nonzero compact operator. Let $A$ be generated by $1, A$ and $T$ (or by $\{A\}^{\prime}$ and $T$ ). Then if $C_{T}=T A-A T$ is so "small" that $C_{T} \in \operatorname{rad} A$, then $A$ has a nontrivial invariant (hyperinvariant) subspace.

PROOF. This is obvious by Theorem 3 and Lomonosov's lemma [1].

Let $A$ be quasinilpotent. Let $A$ be the subalgebra generated by $A$ and 1 , or by $\{A\}^{\prime}$. Then $\operatorname{rad} A \neq\{0\}$.

Question. Is there any nonzero compact operator $T$ such that the subalgebra $R$ generated by $A$ and $T$ has $\operatorname{rad}(R) \neq 0$ ? 


\section{REFERENCES}

1. V. I. Lomonosov, Invariant subspaces for the family of operators which commutes with a completely continuous operator, Functional Anal. Appl. 7 (1973), 213-214.

2. J. Daughtry, An invariant subspace theorem, Proc. Amer. Math. Soc. 49 (1975), 267-268.

3. H. W. Kim, Carl Pearcy and A. L. Shields, Rank one commutator and hyperinvariant subspaces, Michigan Math. J. 22 (1976), 193-194.

4. P. R. Halmos, A Hilbert space problem book, Problem 184, Van Nostrand-Reinhold, Princeton, N. J., 1967.

Department of Mathematics, Purdue University, West lafayette, Indiana 47907 